MATHEMATICS OF COMPUTATION

Volume 74, Number 249, Pages 363-381

S 0025-5718(04)01658-8

Article electronically published on March 23, 2004

\title{
MAASS CUSP FORMS FOR LARGE EIGENVALUES
}

\author{
HOLGER THEN
}

\begin{abstract}
We investigate the numerical computation of Maaß cusp forms for the modular group corresponding to large eigenvalues. We present Fourier coefficients of two cusp forms whose eigenvalues exceed $r=40000$. These eigenvalues are the largest that have so far been found in the case of the modular group. They are larger than the 130millionth eigenvalue.
\end{abstract}

\section{INTRODUCTION}

To extend the classical theory of Dirichlet series with Euler products, Maaß Maa49 studied nonanalytic automorphic functions, nowadays called Maaß waveforms. They are defined in the upper half-plane,

$$
\mathcal{H}=\{z=x+\mathrm{i} y ; x, y \text { real, } y>0\}
$$

equipped with the hyperbolic metric

$$
d s^{2}=\frac{d x^{2}+d y^{2}}{y^{2}} .
$$

Maaß waveforms $f$ are real analytic eigenfunctions of the hyperbolic Laplacian,

$$
(\Delta+\lambda) f(z)=0 .
$$

The Laplacian in the hyperbolic metric reads

$$
\Delta=y^{2}\left(\frac{\partial^{2}}{\partial x^{2}}+\frac{\partial^{2}}{\partial y^{2}}\right)
$$

and is invariant under the group of linear fractional transformations

$$
z \mapsto \gamma z=\frac{a z+b}{c z+d} ; \quad a, b, c, d \text { real, } \quad a d-b c=1 .
$$

This group is isomorphic to the group of matrices

$$
\gamma=\left(\begin{array}{ll}
a & b \\
c & d
\end{array}\right) \in \mathrm{SL}(2, \mathbb{R}) /\{ \pm 1\}
$$

In addition, Maaß waveforms are required to satisfy the automorphy condition

$$
f(\gamma z)=f(z) \quad \forall \gamma \in \Gamma
$$

Received by the editor November 26, 2002 and, in revised form, July 4, 2003.

2000 Mathematics Subject Classification. Primary 11F72, 11F30; Secondary 11F12, 11Yxx, 11-4, 81Q50.

Key words and phrases. Automorphic forms, spectral theory, computational number theory, Fourier coefficients, explicit machine computation, multiplicative number theory, Hecke operators, Ramanujan-Petersson conjecture, Sato-Tate conjecture, quantum chaos, Berry conjecture, approximation of special functions, modified Bessel function. 
relative to a cofinite discrete subgroup

$$
\Gamma \subset \mathrm{SL}(2, \mathbb{R}) /\{ \pm 1\}
$$

and to satisfy the bound

$$
f(z)=O\left(y^{\kappa}\right) \quad \text { for } y \rightarrow \infty
$$

uniformly in $x$ for some positive constant $\kappa$ and similarly in the other cusps. Maaß waveforms which vanish in all the cusps, i.e., for which

$$
f(z) \rightarrow 0 \quad \text { as } \quad \Im z \rightarrow+\infty
$$

and analogously at the other cusps are called Maaß cusp forms. For references, cf., e.g., Sel56, Roe66, Hej83, Ter85, Miy89, Ven90, Iwa95.

We choose the discrete group $\Gamma$ to be the modular group,

$$
\Gamma=\operatorname{PSL}(2, \mathbb{Z})=\operatorname{SL}(2, \mathbb{Z}) /\{ \pm 1\} .
$$

It is generated by two elements

$$
\left(\begin{array}{ll}
1 & 1 \\
0 & 1
\end{array}\right), \quad\left(\begin{array}{cc}
0 & -1 \\
1 & 0
\end{array}\right),
$$

which are isomorphic to the translation and the inversion

$$
z \mapsto z+1, \quad z \mapsto-\frac{1}{z},
$$

and has a fundamental domain which can be chosen to be

$$
\mathcal{F}=\Gamma \backslash \mathcal{H}=\left\{z=x+\mathrm{i} y \in \mathcal{H} ; \quad|x|<\frac{1}{2}, \quad|z|>1\right\} .
$$

Maaß cusp forms are square integrable over the fundamental domain

$$
\int_{\mathcal{F}}|f(z)|^{2} d \mu<\infty
$$

where the volume element is

$$
d \mu=\frac{d x d y}{y^{2}} .
$$

The reflection symmetry of the fundamental domain $\mathcal{F}$ in the line $x=0$ implies that the Maaß waveforms can be chosen such that they fall into two symmetry classes, the even functions $f(x+\mathrm{i} y)=f(-x+\mathrm{i} y)$ and the odd functions $f(x+\mathrm{i} y)=$ $-f(-x+\mathrm{i} y)$, respectively. From the definition of Maaß waveforms (1.1), (1.2), and (1.3), it follows that they can be expanded into Fourier series,

$$
f(z)=u_{0}(y)+\sum_{n \in \mathbb{N}} a_{n} y^{\frac{1}{2}} K_{\mathrm{i} r}(2 \pi n y) \operatorname{cs}(2 \pi n x),
$$

where

$$
u_{0}(y)= \begin{cases}b_{0} y^{\frac{1}{2}+\mathrm{i} r}+b_{1} y^{\frac{1}{2}-\mathrm{i} r} & \text { if } r \neq 0, \\ b_{2} y^{\frac{1}{2}}+b_{3} y^{\frac{1}{2}} \ln y & \text { if } r=0\end{cases}
$$

and

$$
\operatorname{cs}(x)= \begin{cases}2 \cos (x) & \text { for the even Maaß waveforms } \\ 2 \sin (x) & \text { for the odd ones. }\end{cases}
$$


$K_{\mathrm{i} r}(x)$ is the $K$-Bessel function (see Appendix $\mathrm{A}$ ) whose order is connected with the eigenvalue $\lambda$ by

$$
\lambda=r^{2}+\frac{1}{4} .
$$

While keeping in mind that $\lambda$ is the true eigenvalue, we will often call $r$ to be the eigenvalue instead.

According to the Roelcke-Selberg spectral resolution of the Laplacian Sel56. Roe66], its spectrum contains both a discrete and a continuous part. The discrete part of the spectrum is spanned by the constant eigenfunction $f_{0}$ and a countable number of Maaß cusp forms $f_{1}, f_{2}, f_{3}, \ldots$ which we take to be ordered with increasing eigenvalues $0=\lambda_{0}<\lambda_{1} \leq \lambda_{2} \leq \lambda_{3} \leq \cdots$. The continuous part of the spectrum $\lambda=r^{2}+\frac{1}{4} \geq \frac{1}{4}$ is spanned by the Eisenstein series $E\left(z, \frac{1}{2}+\mathrm{i} r\right)$ which are known analytically Maa49, Kub73]. The functions $\Lambda\left(\frac{1}{2}+\mathrm{i} r\right) E\left(z, \frac{1}{2}+\mathrm{i} r\right)$ are even and their Fourier coefficients are given by

$$
b_{0}=\Lambda\left(\frac{1}{2}+\mathrm{i} r\right), \quad b_{1}=\Lambda\left(\frac{1}{2}-\mathrm{i} r\right), \quad a_{n}=\sum_{\substack{c, d \in \mathbb{Z} \\ c d=n}}\left|\frac{c}{d}\right|^{\mathrm{i} r},
$$

with

$$
\Lambda(s)=\pi^{-s} \Gamma(s) \zeta(2 s) .
$$

The positive eigenvalues and their associated Maaß cusp forms are not known analytically. Hence one has to calculate them numerically.

References concerning this computational work in the case of the modular group are: Car71, ASD71, Haa77, Car78, Hej81, GS82, HB82, GS84, Sta84, Win88, Hej91, CGS91, Hun91, Sch91, HR92, Hej92a Hej92b, Ste92, HA93, Ste94, Hej99. Ave03. The first breakthrough to go beyond $r=27.284$ was made by Hejhal Hej91 who computed the first 123 eigenvalues and 36 more in three intervals around $r \approx 125, r \approx 250$, and $r \approx 500$, respectively. He used the truncated Fourier expansion

$$
f(z)=\sum_{n=1}^{M} a_{n} y^{\frac{1}{2}} K_{\mathrm{i} r}(2 \pi n y) \operatorname{cs}(2 \pi n x)+[[\varepsilon]]
$$

in the automorphy condition

$$
f(z)=f\left(-\frac{1}{z}\right)
$$

and obtained a linear system of equations,

$$
\sum_{n=2}^{M} a_{n} I_{n}\left(z_{m}\right)=-I_{1}\left(z_{m}\right), \quad z_{m} \in \mathcal{F}, \quad 1 \leq m \leq M-1,
$$

with

$$
\begin{aligned}
I_{n}(z)= & y^{\frac{1}{2}} K_{\mathrm{i} r}(2 \pi n y) \operatorname{cs}(2 \pi n x) \\
& -\left(\Im\left(-\frac{1}{z}\right)\right)^{\frac{1}{2}} K_{\mathrm{i} r}\left(2 \pi n \Im\left(-\frac{1}{z}\right)\right) \operatorname{cs}\left(2 \pi n \Re\left(-\frac{1}{z}\right)\right),
\end{aligned}
$$

where, for suitable $M$, the error term $[[\varepsilon]]$ is of negligible size and can be omitted. After multiplication of this linear system of equations with $\mathrm{e}^{\frac{\pi r}{2}}$, it was solved for 
successive $r$ values on a grid. Eigenvalues were found by checking whether the coefficients are multiplicative,

$$
a_{1}=1, \quad a_{m p}=a_{m} a_{p}-a_{\frac{m}{p}}, \quad p \text { prime, }
$$

with the convention $a \frac{m}{p}=0$ if $p$ does not divide $m$. Because $I_{n}\left(z_{m}\right)$ gets small for large $n$, the linear system of equations is unstable.

An attempt to get around these instabilities was carried out by Stark Sta84, Hejhal and Arno [HA93, and Steil [Ste92, Ste94]. They used the eigenvalue equations

$$
\mathrm{T}_{m} f(z)=t_{m} f(z)
$$

of the Hecke operators

$$
\mathrm{T}_{m} f(z)=\frac{1}{\sqrt{m}} \sum_{\substack{a d=m \\ b \bmod d, d>0}} f\left(\frac{a z+b}{d}\right) ;
$$

see Maaß [Maa49, Maa64]. The Hecke operators $\mathrm{T}_{m}$ are self-adjoint, commute with the Laplacian, with the symmetry of the fundamental domain, and amongst each other. They are multiplicative,

$$
\mathrm{T}_{m} \mathrm{~T}_{n} f(z)=\sum_{\substack{d \mid(m, n) \\ d>0}} \mathrm{~T}_{\frac{m n}{d^{2}}} f(z),
$$

and their eigenvalues are connected with the Fourier coefficients of the Maaß cusp forms by

$$
a_{m}=a_{1} t_{m} .
$$

Normalizing Maaß cusp forms according to $a_{1}=1$, the nonlinear system of equations

$$
\begin{gathered}
\mathrm{T}_{p} f(z)=a_{p} f(z), \\
a_{m p}=a_{m} a_{p}-a_{\frac{m}{p}}, \quad p \text { prime }
\end{gathered}
$$

allowed Steil to compute all eigenvalues up to $r=350$ (4401 even and 4776 odd eigenfunctions) and between $r=500$ and 510 (395 even and 410 odd). He was also able to compute eigenvalues around $r \approx 4000$.

Finally, Hejhal Hej99] found a linear stable algorithm for computing Maaß cusp forms together with their eigenvalues. His algorithm is based on finite Fourier transforms and implicit automorphy and can be applied to holomorphic cusp forms as well as to Maaß cusp forms. Furthermore, his algorithm can also be applied to nonarithmetic groups and can be extended to groups whose fundamental domain has several cusps SS02. With this algorithm, Hejhal found eigenvalues around $r \approx 11000$. The main obstacle to go beyond was a lack of further memory. Our goal in the present paper will be to obtain larger eigenvalues. We keep the main ideas, but we optimize the algorithmic procedure used in finding the eigenvalues. Furthermore, we make careful use of the memory. This enables us to compute eigenvalues up to $r \approx 40000$. Limitations to go beyond this are due not to lack of memory, but, rather to CPU time. The latter (which scales with the third power of $r$ ) exceeds four weeks on a $750 \mathrm{MHz}$ SUN UltraSPARC-III processor. Currently, the only "larger" Maaß cusp forms available on the numerical front are those explored 
by Hejhal and Strömbergsson HS01] in their recent work with waveforms of CMtype, i.e., waveforms on congruence subgroups which arise as lifts of automorphic forms on $\mathrm{GL}(1)$.

\section{HeJHAL'S ALGORITHM}

We make use of Hejhal's algorithm [Hej99], which uses the Fourier expansion (1.5) and the automorphy condition (1.2). In the present paper, we restrict ourselves to the modular group $\Gamma=\operatorname{PSL}(2, \mathbb{Z})$ which is generated by the translation $z \mapsto z+1$ and the inversion $z \mapsto-\frac{1}{z}$. There do not exist small eigenvalues $0<\lambda=r^{2}+\frac{1}{4} \leq \frac{1}{4}$ for the modular group; see [Roe66]. Therefore, $r$ is real and the term $u_{0}(y)$ in the Fourier expansion of Maaß cusp forms (1.4) vanishes. Due to the exponential decay of the $K$-Bessel function for large arguments (A.1) and the bound

$$
\left|a_{n}\right| \leq d(n) n^{\frac{1}{4}}
$$

for the coefficients (see [Vig83), where $d(n)$ counts the number of divisors of $n$, the absolutely convergent Fourier expansion can be truncated anytime we bound $y$ from below. Given $\varepsilon>0, r$, and $y$, we determine the smallest $M=M(\varepsilon, r, y)$ such that the inequalities

$$
2 \pi M y \geq r \quad \text { and } \quad K_{\mathrm{i} r}(2 \pi M y) \leq \varepsilon \max _{x}\left(K_{\mathrm{i} r}(x)\right)
$$

hold. Larger $y$ allow smaller $M$. In all the truncated terms, i.e., within

$$
[[\varepsilon]]=\sum_{n=M+1}^{\infty} a_{n} y^{\frac{1}{2}} K_{\mathrm{i} r}(2 \pi n y) \operatorname{cs}(2 \pi n x)
$$

the $K$-Bessel function decays exponentially in $n$, and already the $K$-Bessel function of the first truncated summand is smaller than $\varepsilon$ times most of the $K$-Bessel functions in the sum of (1.5). Thus, the error $[[\varepsilon]]$ does at most marginally exceed $\varepsilon$. The reason for why $[[\varepsilon]]$ can exceed $\varepsilon$ somewhat is due to the possibility that the summands in (1.5) can cancel each other and that the first few coefficients $a_{n}$ in the truncated terms may occasionally be much bigger than in (1.5)).

By a finite Fourier transform, the Fourier expansion (1.5) is solved for its coefficients

$$
a_{m} y^{\frac{1}{2}} K_{\mathrm{i} r}(2 \pi m y)=\frac{1}{2 Q} \sum_{x \in \mathbb{X}} f(x+\mathrm{i} y) \operatorname{cs}(-2 \pi m x)+[[\varepsilon]],
$$

where $\mathbb{X}$ is an equidistributed set of $Q$ numbers,

$$
\mathbb{X}=\left\{\frac{\frac{1}{2}}{2 Q}, \frac{\frac{3}{2}}{2 Q}, \ldots, \frac{Q-\frac{3}{2}}{2 Q}, \frac{Q-\frac{1}{2}}{2 Q}\right\},
$$

with $2 Q>M+m$.

By automorphy we have

$$
f(z)=f\left(z^{*}\right)
$$

where $z^{*}$ is the $\Gamma$-pullback of the point $z$ into the fundamental domain $\mathcal{F}$,

$$
z^{*}=\gamma z, \quad \gamma \in \Gamma, \quad z^{*} \in \mathcal{F} .
$$

Any Maaß cusp form can thus be approximated by

$$
f(x+\mathrm{i} y)=f\left(x^{*}+\mathrm{i} y^{*}\right)=\sum_{n=1}^{M_{0}} a_{n} y^{* \frac{1}{2}} K_{\mathrm{i} r}\left(2 \pi n y^{*}\right) \operatorname{cs}\left(2 \pi n x^{*}\right)+[[\varepsilon]],
$$


where $y^{*}$ is always larger than or equal to the height $y_{0}$ of the lowest points in the fundamental domain $\mathcal{F}$,

$$
y_{0}=\min _{z \in \mathcal{F}}(y)=\frac{\sqrt{3}}{2},
$$

effectively allowing us to replace $M(\varepsilon, r, y)$ by $M_{0}=M\left(\varepsilon, r, y_{0}\right)$.

Choosing $y$ smaller than $y_{0}$, the $\Gamma$-pullback of any point into the fundamental domain $\mathcal{F}$ makes use at least once of the inversion $z \mapsto-\frac{1}{z}$, possibly together with the translation $z \mapsto z+1$. This is called implicit automorphy, since it guarantees the invariance $f(z)=f\left(-\frac{1}{z}\right)$, whereas the condition $f(z)=f(z+1)$ is satisfied by the Fourier expansion.

Making use of the implicit automorphy by replacing $f(x+\mathrm{i} y)$ in (2.1) with the right-hand side of (2.2) yields

$$
\begin{aligned}
& a_{m} y^{\frac{1}{2}} K_{\mathrm{i} r}(2 \pi m y) \\
& \quad=\frac{1}{2 Q} \sum_{x \in \mathbb{X}} \sum_{n=1}^{M_{0}} a_{n} y^{* \frac{1}{2}} K_{\mathrm{i} r}\left(2 \pi n y^{*}\right) \operatorname{cs}\left(2 \pi n x^{*}\right) \operatorname{cs}(-2 \pi m x)+[[2 \varepsilon]]
\end{aligned}
$$

for $1 \leq m \leq M$, which is the central identity in the algorithm. With this identity, the coefficients $a_{m}$ can be determined for all $m$ so long as $y<y_{0}$ is chosen such that $K_{\mathrm{i} r}(2 \pi m y)$ does not become too small.

Taking $1 \leq m \leq M_{0}$ and forgetting about the error [[2ع]], the set of equations can be rewritten as

$$
\sum_{n=1}^{M_{0}} V_{m n}(r, y) a_{n}=0, \quad m \geq 1,
$$

where the matrix $V=\left(V_{m n}\right)$ is given by

$$
\begin{aligned}
V_{m n}(r, y)=y^{\frac{1}{2}} & K_{\mathrm{i} r}(2 \pi m y) \delta_{m n} \\
& -\frac{1}{2 Q} \sum_{x \in \mathbb{X}} y^{* \frac{1}{2}} K_{\mathrm{i} r}\left(2 \pi n y^{*}\right) \operatorname{cs}\left(2 \pi n x^{*}\right) \operatorname{cs}(-2 \pi m x) .
\end{aligned}
$$

Since $y$ can always be chosen such that $K_{\mathrm{ir}}(2 \pi m y)$ is not too small, the diagonal terms in the matrix $V$ do not vanish for large $m$ and the matrix is well conditioned. This makes the algorithm stable.

We are now looking for nontrivial solutions of 2.3 with $1 \leq m \leq M_{0}$ that simultaneously give the eigenvalues $r$ and the coefficients $a_{n}$. Trivial solutions are avoided by setting $a_{1}=1$; cf. Miy89, assertions 4.5.16 and 4.6.11].

Since the eigenvalues $r$ are unknown, we discretize the $r$ axis and solve for each $r$ value on this grid

$$
\sum_{n=2}^{M_{0}} V_{m n}\left(r, y^{\# 1}\right) a_{n}=-V_{m 1}\left(r, y^{\# 1}\right), \quad 1 \leq m \leq M_{0}-1,
$$

where $y^{\# 1}<y_{0}$ is chosen such that $K_{\mathrm{i} r}\left(2 \pi m y^{\# 1}\right)$ is not too small for $1 \leq m \leq$ $M_{0}-1$. A good value to try for $y^{\# 1}$ is given by

$$
2 \pi M_{0} y^{\# 1}=r .
$$

Hejhal Hej99 solves (2.4) a second time with a different $y^{\# 2}$ and checks whether the coefficients are independent of the choice of $y$. 
Some words have to be said about what we mean by solving the inhomogeneous system (2.4), since it may happen that there is not always a solution unless $r$ is an eigenvalue. By solving a linear inhomogeneous system of equations

$$
A x=y,
$$

we mean that we compute

$$
x=\tilde{A}^{-1} y
$$

where $\tilde{A}^{-1}$ is determined such that $\tilde{A}^{-1} A$ is a diagonal matrix where as many diagonal elements as possible are equal to one.

\section{Some Improvements}

We restructure Hejhal's algorithm Hej99 in the way it finds the eigenvalues. Instead of solving (2.4) a second time, we check whether the coefficients $a_{n}=a_{n}^{\# 1}$ obtained actually solve (2.3) by computing

$$
g_{m}=\sum_{n=1}^{M_{0}} V_{m n}\left(r, y^{\# 2}\right) a_{n}^{\# 1}, \quad 1 \leq m \leq M_{0},
$$

where $y^{\# 2}=\frac{9}{10} y^{\# 1}$ is a good choice for an independent $y$ value. Only if all $g_{m}$ vanish simultaneously can the given $r$ be an eigenvalue and the computed $a_{n}$ 's the Fourier coefficients of a Maaß cusp form.

The probability of finding an $r$ value such that all $g_{m}$ vanish simultaneously is zero because the discrete eigenvalues are of measure zero in the real numbers. Therefore, we make use of the intermediate value theorem. We let $r$ run through a grid of discretized $r$ values and look for simultaneous changes of sign in the $g_{m}$.

It is conjectured [BGGS92, BSS92, Bol93, Sar95] BLS96] that the eigenvalues of the Laplacian for even and odd cusp forms each possess a spacing distribution close to that of a Poisson random process. One therefore expects that small spacings will occur comparably often (due to level clustering). In order not to miss eigenvalues which lie close together, we have to make sure that at least one point of the $r$ grid lies between any two successive eigenvalues. On the other hand, we do not want to waste CPU time if there are large spacings. Therefore, we use an adaptive algorithm which tries to predict the next best $r$ value of the grid. It is based on the observation that the coefficients $a_{n}$ of two Maaß cusp forms of successive eigenvalues must differ. Assume that two eigenvalues lie close together and that the coefficients of the two Maaß cusp forms do not differ much. Numerically then both Maaß cusp forms would tend to be similar - which contradicts the fact that different Maaß cusp forms are orthogonal to each other with respect to the Petersson scalar product

$$
\left\langle f_{i}, f_{j}\right\rangle=\int_{\mathcal{F}} \overline{f_{i}(z)} f_{j}(z) \frac{d x d y}{y^{2}}=0, \quad \text { if } \lambda_{i} \neq \lambda_{j} .
$$

Maaß cusp forms corresponding to different eigenvalues are orthogonal because the Laplacian is an essentially self-adjoint operator. Thus, if successive eigenvalues lie close together, the coefficients $a_{n}$ must change fast when varying $r$. In contrast, if successive eigenvalues are separated by large spacings numerically, it turns out that often the coefficients change only slowly upon varying $r$. Defining

$$
\tilde{a}_{n}=\frac{a_{n}}{\sqrt{\sum_{m=1}^{M_{0}}\left|a_{m}\right|^{2}}}, \quad 1 \leq n \leq M_{0},
$$


our adaptive algorithm predicts the next $r$ value of the grid such that the change in the coefficients is

$$
\sum_{n=1}^{M_{0}}\left|\tilde{a}_{n}\left(r_{\text {old }}\right)-\tilde{a}_{n}\left(r_{\text {new }}\right)\right|^{2} \approx 0.04 .
$$

For this prediction, the last step in the $r$ grid together with the last change in the coefficients is used to extrapolate linearly the choice for the next $r$ value of the grid.

However the adaptive algorithm is not rigorous. Sometimes the prediction of the next $r$ value fails so that it is too close or too far away from the previous one. A small number of small steps does not bother us unless the step size tends to zero. But, if the step size is too large, such that the left-hand side of (3.1) exceeds 0.16 , we reduce the step size and try again with a smaller $r$ value.

Compared to earlier algorithms, our adaptive one tends to miss significantly fewer eigenvalues per run.

We are searching for simultaneous sign changes in the quantities $g_{m}$. Once we have found such in at least half of all the $g_{m}$ 's, we have found an interval $\left[r_{\text {old }}, r_{\text {new }}\right]$ which contains an eigenvalue $r$ with high probability. The next step is to check whether this interval really contains an eigenvalue, and, if so, to find this eigenvalue by some interpolation or bisection.

In fact, we use a trisection which is based on a bisection together with a Newtonian interpolation. One first bisects the interval $\left[r_{\text {old }}, r_{\text {new }}\right]$ and re-examines the sign changes. The interval with the most is then divided further by Newtonian interpolation, which ensures fast convergence. In the next step of the trisection, we again examine the sign changes and highlight that interval which contains the most simultaneous sign changes in the $g_{m}$ 's. If there is an eigenvalue contained in the successive intervals of the trisection, the number of $g_{m}$ 's that simultaneously change their sign increases from step to step in the iteration until the size of the interval approaches zero and the eigenvalue is found. In the opposite case, the number of $g_{m}$ 's which simultaneously change their sign decreases from step to step in the iteration until one suspects that there is no eigenvalue contained in the interval $\left[r_{\text {old }}, r_{\text {new }}\right]$.

\section{RESUlts}

After some preliminary tests of our algorithm, where we computed some eigenvalues of the odd symmetry class around $r \approx 10000$ (see Table 1 ) and two larger eigenvalues $r=20000.00164526$ (even) and $r=20000.00020183$ (odd), we decided to compute two Maaß cusp forms corresponding to eigenvalues $r \approx 40000$, one for each symmetry class. For the size of the error in truncating the Fourier expansion, we chose $\varepsilon=10^{-7}$, which we took also for the accuracy of our $K$-Bessel function. For this cutoff, we had to take 7395 Fourier coefficients into account of which 938 have prime index. Finding the two eigenvalues together with their Maaß cusp forms took four weeks of CPU time for each on a $750 \mathrm{MHz}$ SUN UltraSPARC-III processor, and 1.3 GB of memory were needed.

Starting at $r=40000$ in the upwards direction, the first even eigenvalue was found at $r=40000.0000916$; the first odd one was found at $r=40000.0001644$. In Tables 2 and 3 we list the first few Fourier coefficients of these two forms. We checked the accuracy of our results with the aid of the multiplicative relations (1.6). The left-hand side of the multiplicative relations coincides with the right-hand side 
TABLE 1 . Eigenvalues of the Maaß cusp forms around $r \approx 10000$ (odd symmetry).

\begin{tabular}{c}
$r$ \\
\hline 10000.00203541 \\
10000.00469659 \\
10000.00735313 \\
10000.00773954 \\
10000.00805085 \\
10000.00947268 \\
10000.01012235 \\
10000.01102222 \\
10000.01373844 \\
10000.01460515 \\
10000.01610617 \\
\hline
\end{tabular}

TABLE 2. The first 174 Fourier coefficients of the Maaß cusp form corresponding to the eigenvalue $r=40000.0000916$ (even symmetry).

\begin{tabular}{rrrrrr}
$a_{1 \cdots 29}$ & $a_{30 \cdots 58}$ & $a_{59 \cdots 87}$ & $a_{88 \cdots 116}$ & $a_{117 \cdots 145}$ & $a_{146 \cdots 174}$ \\
\hline 1 & -0.2674 & 0.5078 & -0.0963 & 0.2399 & 1.3396 \\
1.2094 & 1.4654 & -0.1024 & 1.1918 & 0.6143 & 0.0787 \\
-0.1799 & -0.8607 & -0.5538 & -1.4379 & 0.2756 & 0.6445 \\
0.4629 & -0.0267 & 1.7726 & 0.1861 & 0.1435 & -1.0355 \\
1.2285 & -0.4445 & 0.7256 & -0.6502 & -0.9778 & -0.1108 \\
-0.2176 & -0.9213 & 0.2074 & -0.2637 & -0.6697 & -0.6101 \\
-0.7499 & -0.4479 & -0.3046 & 1.1780 & -0.1000 & 0.7014 \\
-0.6494 & 1.3920 & -0.0322 & -1.3269 & 0.6785 & 0.3556 \\
-0.9675 & -1.3062 & -0.6666 & 0.1549 & -0.6027 & -0.1347 \\
1.4858 & 0.0446 & -0.1701 & -0.9765 & 0.8777 & 1.8005 \\
0.1485 & -0.7980 & 0.2527 & -0.5292 & 1.6805 & 0.0207 \\
-0.0833 & 0.5559 & -1.1143 & -0.1436 & 1.1116 & -0.5205 \\
-0.2480 & 0.1632 & 1.2144 & 0.2360 & -0.2161 & 1.2166 \\
-0.9070 & 1.2010 & 0.6285 & -0.3695 & -0.3686 & 0.0880 \\
-0.2211 & 0.0687 & 1.1076 & 0.0800 & 1.5360 & -1.0575 \\
-1.2484 & -1.1888 & 1.6837 & -0.5686 & -0.0123 & 1.0532 \\
-0.3674 & -1.6986 & -0.0916 & 0.1612 & 0.8099 & 1.0933 \\
-1.1703 & 0.9739 & -0.5000 & 0.1658 & -0.8063 & -0.8840 \\
-1.0799 & 0.2246 & -0.1113 & -0.5918 & 0.4349 & 0.2573 \\
0.5687 & -0.4376 & 0.0540 & 0.7798 & 0.2387 & -0.0329 \\
0.1349 & 0.6161 & 1.0058 & 0.1639 & 1.3926 & -2.0562 \\
0.1795 & 0.0661 & -1.5339 & 0.8569 & 0.3056 & -0.3518 \\
-1.4044 & -0.1149 & 0.9039 & 0.2205 & -1.0796 & -0.0877 \\
0.1169 & -0.4892 & 0.6724 & -0.2505 & -0.4266 & -0.9383 \\
0.5094 & 0.4282 & -1.6999 & 0.9363 & -0.1751 & -0.5461 \\
-0.3000 & 0.1824 & 0.0624 & 1.7346 & 1.4690 & 1.0451 \\
0.3540 & 0.4871 & -0.4514 & 0.2351 & -0.0367 & 0.5561 \\
-0.3473 & 0.1943 & 1.4526 & -1.7254 & 1.2081 & -0.4913 \\
0.2914 & 0.3524 & -0.0524 & 0.1349 & 0.3580 & -0.0634 \\
\hline & & & & &
\end{tabular}


TABLE 3. The first 174 Fourier coefficients of the Maaß cusp form corresponding to the eigenvalue $r=40000.0001644$ (odd symmetry).

\begin{tabular}{rrrrrr}
$a_{1 \cdots 29}$ & $a_{30 \cdots 58}$ & $a_{59 \cdots 87}$ & $a_{88 \cdots 116}$ & $a_{117 \cdots 145}$ & $a_{146 \cdots 174}$ \\
\hline 1 & -0.1394 & 0.9151 & 1.2840 & -0.1093 & 0.6031 \\
-0.5454 & 0.7975 & -0.1794 & -1.6376 & -0.4991 & -0.7992 \\
0.8637 & -1.0355 & -1.6485 & 0.0410 & 0.4208 & 0.6505 \\
-0.7026 & 1.1944 & -0.4350 & 0.1176 & 0.2371 & 0.5716 \\
0.2957 & -0.8396 & -0.0695 & 0.1591 & 0.9124 & 0.4298 \\
-0.4711 & 0.0809 & 0.3686 & 0.6888 & 0.8991 & -1.6099 \\
0.2733 & 0.1785 & 0.1271 & 0.0114 & -1.5822 & 1.4495 \\
0.9285 & -0.9259 & -0.6514 & 0.4616 & -0.5604 & -0.3911 \\
-0.2540 & -0.8514 & 1.1840 & -0.8943 & -0.5655 & -0.2062 \\
-0.1612 & 0.3713 & -1.0815 & 1.8048 & 0.0378 & 0.2358 \\
1.3829 & 0.2746 & -0.1956 & 0.5047 & -1.0520 & -0.2609 \\
-0.6068 & -1.8319 & -0.0441 & -0.3512 & 0.8345 & -0.6977 \\
0.4299 & -0.1287 & 1.8049 & 0.6412 & -1.1665 & -0.3762 \\
-0.1491 & -1.3506 & -0.2359 & -0.0862 & -0.0694 & 1.2012 \\
0.2554 & -0.9716 & -1.1057 & -0.7252 & -0.6646 & -0.3062 \\
0.1962 & -0.0751 & 0.5050 & 0.4610 & -0.8391 & -0.0619 \\
1.5395 & 0.1235 & -0.7882 & 0.3993 & 0.4267 & 0.3716 \\
0.1386 & -0.0211 & -1.0967 & 0.0698 & -0.6457 & -1.0045 \\
1.5611 & 0.1694 & 0.3780 & -0.7585 & -0.3202 & 1.2870 \\
-0.2077 & -0.9253 & -0.2025 & -1.8158 & 1.4295 & 0.3531 \\
0.2361 & 0.4977 & 0.6896 & 0.7609 & 0.5571 & -0.3239 \\
-0.7542 & 1.3296 & 0.0580 & -0.2113 & 0.1068 & -0.1377 \\
-0.2266 & -0.3019 & -0.6815 & -0.2230 & 0.2784 & 0.2192 \\
0.8019 & 1.3908 & 0.9990 & -0.7997 & -0.0568 & -0.8151 \\
-0.9126 & 0.5907 & 0.5937 & 0.0536 & -0.0182 & -0.2483 \\
-0.2344 & 0.4089 & -0.1658 & 1.7004 & -0.9844 & -0.3966 \\
-1.0831 & 0.2538 & 0.4552 & -0.7353 & 0.5945 & 0.9489 \\
-0.1921 & 1.3483 & 0.7366 & -0.0670 & -0.0499 & -0.3371 \\
-0.3333 & 0.1817 & -0.2879 & 0.2341 & -0.0985 & 0.1570 \\
\hline & & & & &
\end{tabular}

up to a discrepancy of size $10^{-3}$. This means that the coefficients are only accurate to three digits. This is much worse than the initially intended accuracy $\varepsilon=10^{-7}$. The reason for this loss of accuracy is that we have computed the eigenvalues only up to an accuracy of twelve digits. Minimal deviations of the eigenvalue $r$ lead to big changes in the coefficients $a_{n}$. But we cannot compute the eigenvalue much more accurately without increasing the accuracy of our $K$-Bessel routine and taking more coefficients in the Fourier expansion into account.

A different check of the accuracy of the results can be done by computing the coefficients $a_{n}$ a second time, with $y^{\# 1}$ replaced by $y^{\# 2}$. But, with this check, one has to be careful because the coefficients may vary less than the size of their actual error. We did this check and found that the coefficients differ in the sixth digit when $y^{\# 1}$ is replaced by $y^{\# 2}$.

All coefficients which we have computed satisfy the Ramanujan-Petersson conjecture

$$
\left|a_{p}\right| \leq 2 \text { for all primes } p \text {. }
$$



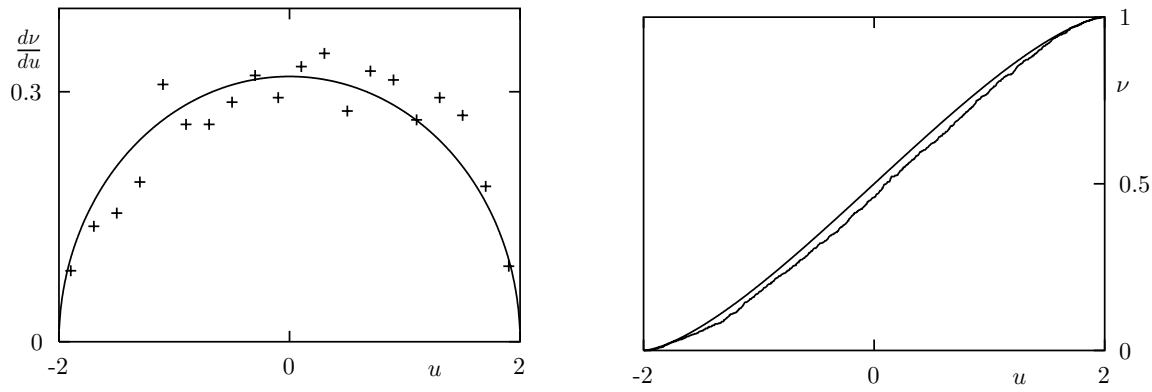

Figure 1. Statistics of the first 938 prime Fourier coefficients of the Maaß cusp form corresponding to the eigenvalue $r=$ 40000.0000916. In the left figure the distribution of the prime coefficients is rendered with points. The solid line is the conjectured semicircle. In the right figure the crumpled line is the integrated distribution of the prime coefficients, and the smooth line is the integrated semicircle.
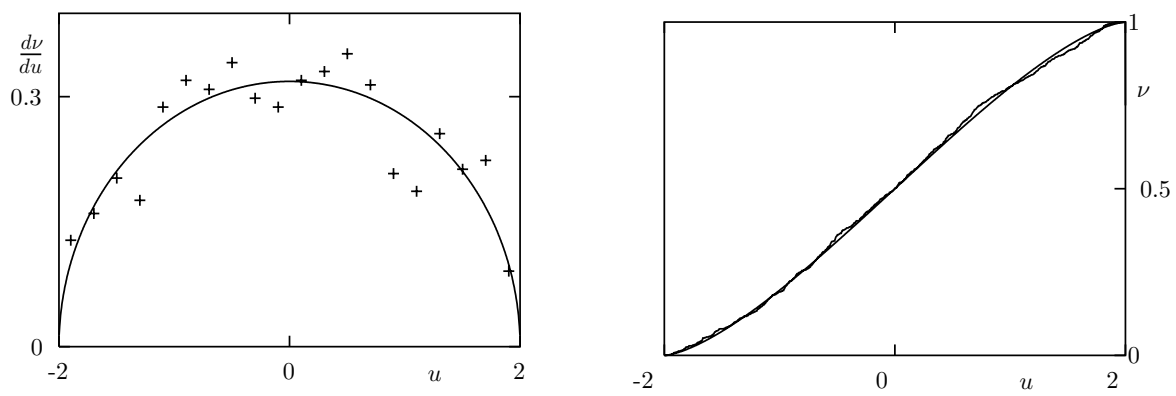

Figure 2. Statistics of the first 938 prime Fourier coefficients of the Maaß cusp form corresponding to the eigenvalue $r=$ 40000.0001644. In the left figure the distribution of the prime coefficients is rendered with points. The solid line is the conjectured semicircle. In the right figure the crumpled line is the integrated distribution of the prime coefficients, and the smooth line is the integrated semicircle.

If the Sato-Tate conjecture is true, the prime coefficients $a_{p}$ of each Maaß cusp form are distributed according to the semicircle law

$$
d \nu(u)= \begin{cases}\frac{1}{2 \pi} \sqrt{4-u^{2}} d u & \text { if }|u|<2 \\ 0 & \text { otherwise }\end{cases}
$$

This means that

$$
\lim _{N \rightarrow \infty} \frac{\frac{1}{\#\{p \text { prime; } p \leq N\}} \sum_{\substack{p \leq N \\ p \text { prime }}} \chi_{[a, b]}\left(a_{p}\right)}{\int_{a}^{b} d \nu(u)}=1
$$

holds for any $-\infty<a<b<\infty$, where $\chi_{[a, b]}(u)$ is the indicator function of the interval $[a, b]$. The prime coefficients which we have computed match the Sato-Tate 
conjecture moderately well; see Figures 1 and [2. One expects of course that the Sato-Tate conjecture $i s$ true and that the plots rapidly improve once one takes more coefficients (with $p>M_{0}$ ) into account; cf. [HA93, Ste94].

\section{VAlue Distribution}

It is believed that Maaß cusp forms behave pretty much like random waves. In particular, in the limit of large eigenvalues, $\lambda=r^{2}+\frac{1}{4} \rightarrow \infty$, a conjecture of Berry Ber77. predicts that each Maaß cusp form has a Gaussian value distribution,

$$
d \rho(u)=\frac{1}{\sqrt{2 \pi} \sigma} \mathrm{e}^{-\frac{u^{2}}{2 \sigma^{2}}} d u,
$$
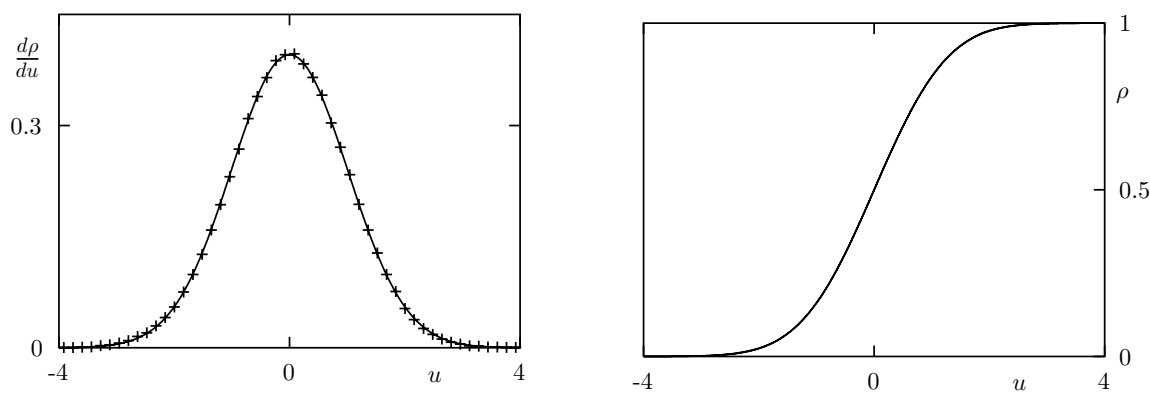

Figure 3. In the left figure the value distribution of the Maaß cusp form corresponding to the eigenvalue $r=40000.0000916$ inside the region $F$ is rendered with points. The solid line is the conjectured Gaussian. In the right figure the solid line is the integrated value distribution of the Maaß cusp form which is indistinguishable from the integrated Gaussian.
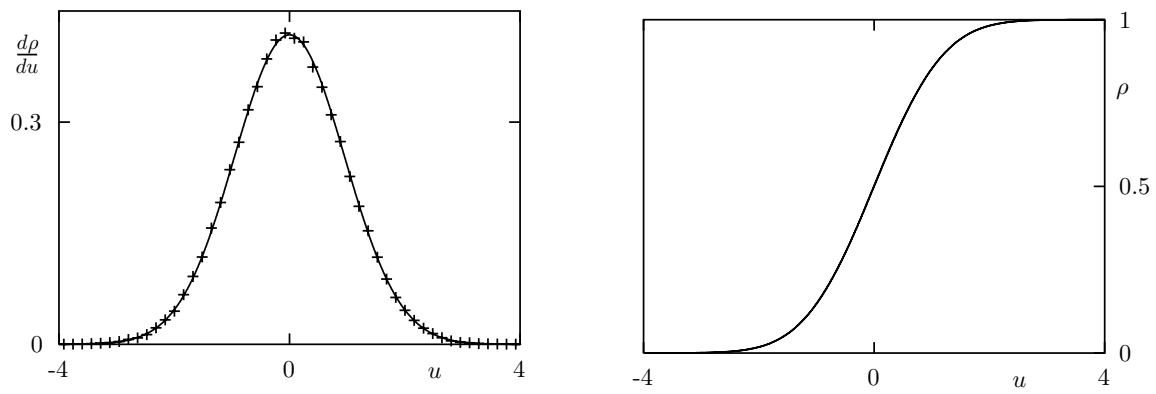

FIGURE 4. In the left figure the value distribution of the Maaß cusp form corresponding to the eigenvalue $r=40000.0001644$ inside the region $F$ is rendered with points. The solid line is the conjectured Gaussian. In the right figure the solid line is the integrated value distribution of the Maaß cusp form which is indistinguishable from the integrated Gaussian. 


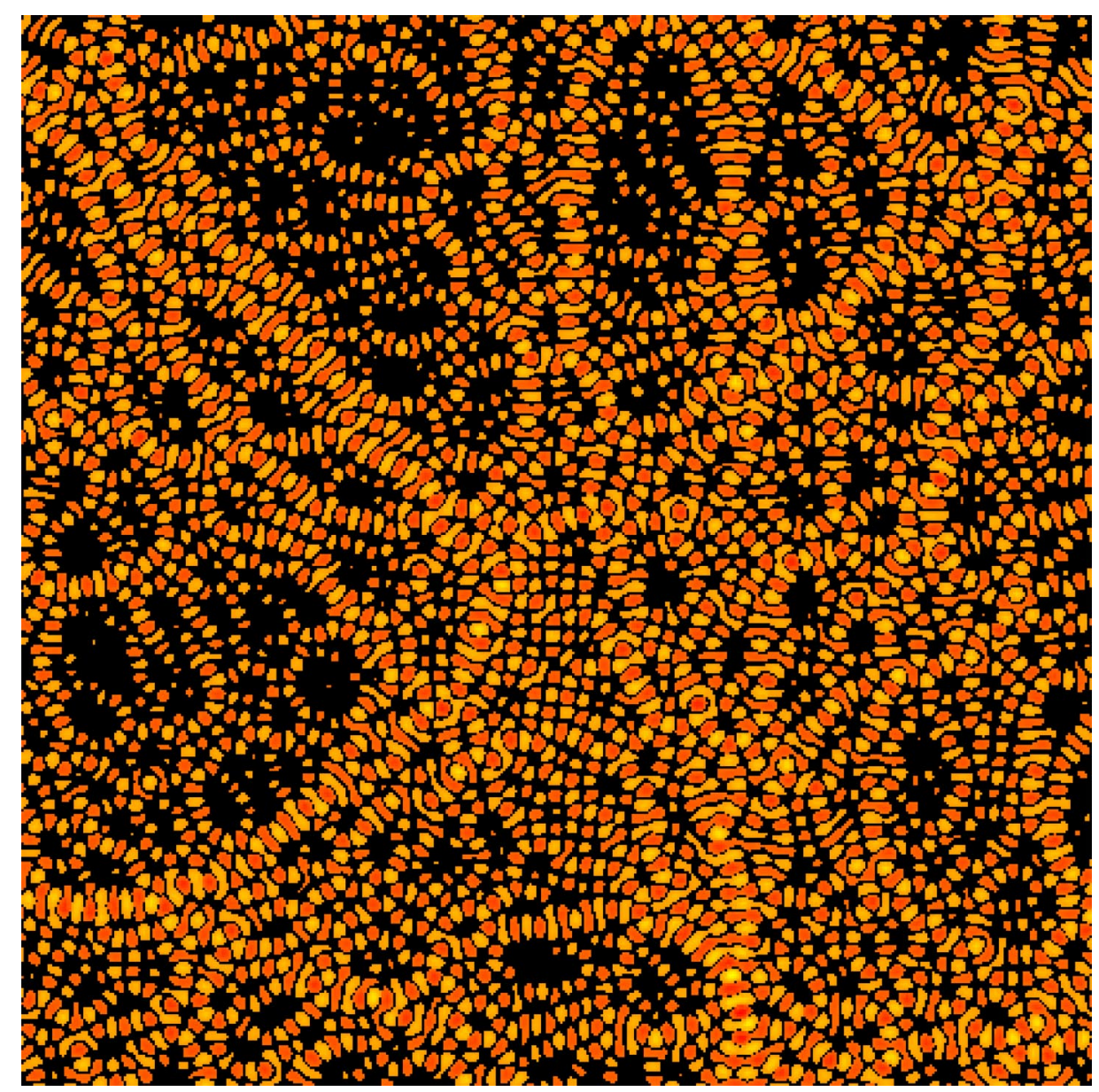

Figure 5. A plot of the Maaß cusp form corresponding to the eigenvalue $r=40000.0000916$ inside the region $F$.

inside any compact regular subregion $F$ of $\mathcal{F}$. This means that

$$
\lim _{\lambda \rightarrow \infty} \frac{\frac{1}{\operatorname{area}(F)} \int_{F} \chi_{[a, b]}(f(z)) d \mu}{\int_{a}^{b} d \rho(u)}=1
$$

holds with variance

$$
\sigma^{2}=\frac{1}{\operatorname{area}(F)} \int_{F}|f(z)|^{2} d \mu
$$

for any $-\infty<a<b<\infty$. Figures 3 and 4 show the value distribution of the Maaß cusp forms corresponding to the eigenvalues $r=40000.0000916$, resp. $r=$ 40000.0001644, inside a small subregion

$$
F=\{z=x+\mathrm{i} y ;-.3 \leq x \leq-.29215,1.1 \leq y \leq 1.10785\}
$$

(see [Hej99, p. 302] for some analogous plots with smaller $r$ ). 


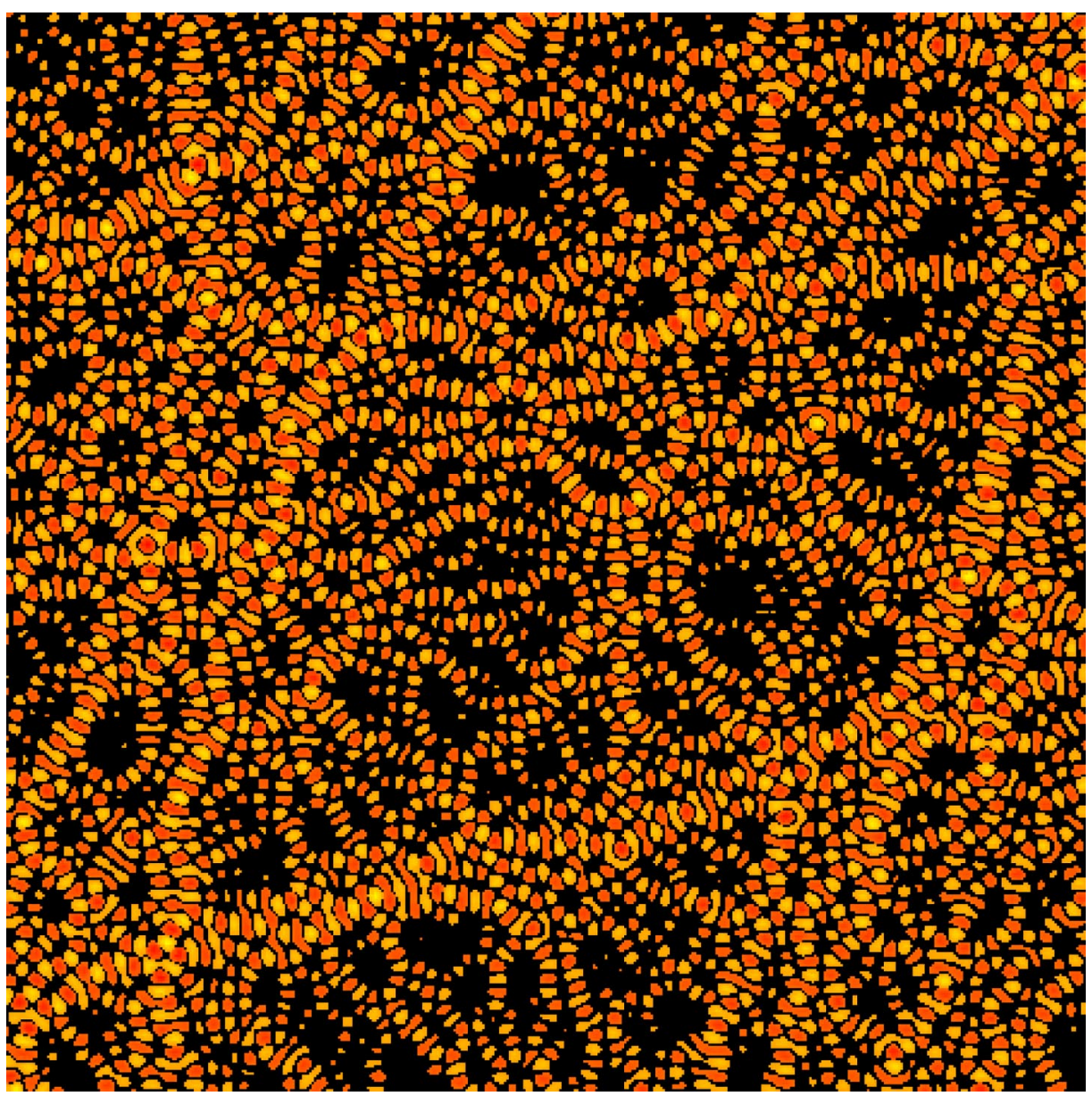

Figure 6. A plot of the Maaß cusp form corresponding to the eigenvalue $r=40000.0001644$ inside the region $F$.

Our numerical data agree well with Berry's conjecture, providing additional numerical evidence that the conjecture does hold. Plots of the two Maaß cusp forms inside the region $F$ are given in Figures 5,6 and Figure 7 shows the small region $F$ inside the fundamental domain $\mathcal{F}$.

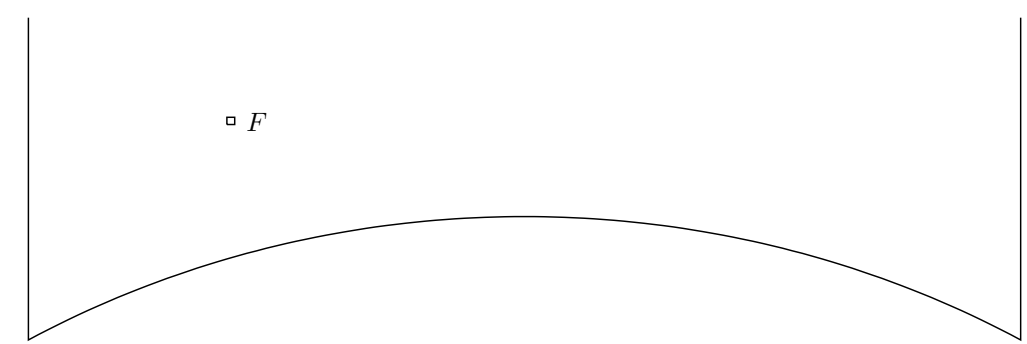

Figure 7. The small subregion $F$ inside the fundamental domain $\mathcal{F}$. 


\section{Appendix A. The $K$-Bessel function}

The $K$-Bessel function is defined by

$$
K_{\mathrm{i} r}(x)=\int_{0}^{\infty} \mathrm{e}^{-x \cosh t} \cos (r t) d t, \quad \Re x>0, \quad r \in \mathbb{C}
$$

(see Watson [Wat44]), and is real for real arguments $x$ and real or imaginary order $\mathrm{i} r$. It satisfies the modified Bessel differential equation

$$
x^{2} u^{\prime \prime}(x)+x u^{\prime}(x)-\left(x^{2}-r^{2}\right) u(x)=0
$$

and decays exponentially for large arguments

$$
K_{\mathrm{i} r}(x) \sim \sqrt{\frac{\pi}{2 x}} \mathrm{e}^{-x} \quad \text { for } x \rightarrow \infty .
$$

A second linearly independent solution of the differential equation is the $I$-Bessel function

$$
I_{\mathrm{i} r}(x)=\left(\frac{x}{2}\right)^{\mathrm{i} r} \sum_{k=0}^{\infty} \frac{\left(\frac{x}{2}\right)^{2 k}}{k ! \Gamma(\mathrm{i} r+k+1)},
$$

which grows exponentially for large arguments

$$
I_{\mathrm{i} r}(x) \sim \sqrt{\frac{1}{2 \pi x}} \mathrm{e}^{x} \quad \text { for } x \rightarrow \infty .
$$

The amplitude of the $K$-Bessel function gets exponentially small if $r$ increases. This can be compensated for by multiplication with the factor $\mathrm{e}^{\frac{\pi r}{2}}$; see Figure 8 .

To compute the $K$-Bessel function numerically, we use asymptotic expansions for large imaginary order, $r \rightarrow \infty$. The most powerful among them is the uniform

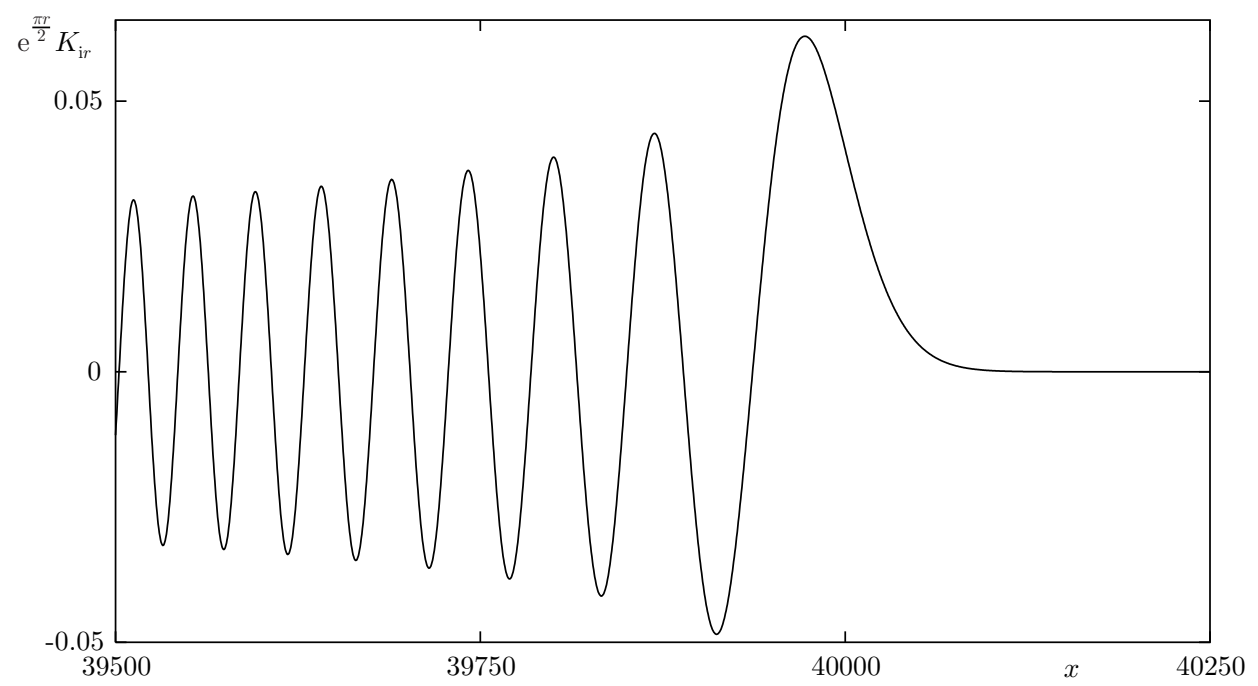

FiguRE 8. $\mathrm{e}^{\frac{\pi r}{2}} K_{\mathrm{i} r}(x)$ for fixed $r=40000$. 
asymptotic expansion

$$
\mathrm{e}^{\frac{\pi r}{2}} K_{\mathrm{i} r}(x) \sim 2^{\frac{1}{2}} \pi\left(\frac{-\xi}{r^{2}-x^{2}}\right)^{\frac{1}{4}}\left(A i(\xi) \sum_{k=0}^{\infty} \frac{A_{k}\left(r^{-\frac{2}{3}} \xi\right)}{r^{2 k}}+A i^{\prime}(\xi) \sum_{k=0}^{\infty} \frac{B_{k}\left(r^{-\frac{2}{3}} \xi\right)}{r^{2 k+\frac{4}{3}}}\right)
$$

where $A i(x)$ and $A i^{\prime}(x)$ denote the Airy function and its derivative, respectively. Here $\xi$ is defined by

$$
\beta=\frac{x}{r}, \quad \gamma=\frac{1}{\sqrt{1-\beta^{2}}}, \quad-\frac{2}{3 r}(-\xi)^{\frac{3}{2}}=\gamma^{-1}-\operatorname{sech}^{-1} \beta,
$$

and the functions $A_{k}(x)$ and $B_{k}(x)$ are given by

$$
\begin{aligned}
& \frac{A_{k}\left(r^{-\frac{2}{3}} \xi\right)}{r^{2 k}}=(-1)^{k} \sum_{s=0}^{2 k} \frac{1+6 s}{1-6 s} \lambda_{s}(-\xi)^{-\frac{3}{2} s} \frac{u_{2 k-s}(\gamma)}{r^{2 k-s}} \\
& \frac{B_{k}\left(r^{-\frac{2}{3}} \xi\right)}{r^{2 k+\frac{4}{3}}}=(-1)^{k+1} \sum_{s=0}^{2 k+1} \lambda_{s}(-\xi)^{-\frac{3}{2} s-\frac{1}{2}} \frac{u_{2 k+1-s}(\gamma)}{r^{2 k+1-s}}
\end{aligned}
$$

where

$$
\lambda_{0}=1, \quad \lambda_{1}=\frac{5}{48}, \quad \lambda_{s}=\frac{(6 s-5)(6 s-1)}{48 s} \lambda_{s-1}, \quad s \geq 2,
$$

and $u_{k}(t)$ are polynomials satisfying the recursion

$$
\begin{aligned}
& u_{0}(t)=1 \\
& u_{k+1}(t)=\frac{1}{2} t^{2}\left(1-t^{2}\right) u_{k}^{\prime}(t)+\frac{1}{8} \int_{0}^{t}\left(1-5 t^{2}\right) u_{k}(t) d t, \quad k \geq 0
\end{aligned}
$$

see, e.g., [Bal66, eq. (2)], [Bal67] eqs. (18), (19), (20)], [CGS91, appendix], GST02. section 5] or compare with [Olv54, eq. (4.24)], CFU57, eq. (6.6)], [AS65, eqs. (9.3.10), (9.3.35), (9.3.40), (9.3.41)]. All terms are real if $x<r$, and using

$$
\frac{2}{3 r} \xi^{\frac{3}{2}}=(-\mathrm{i} \gamma)^{-1}-\sec ^{-1} \beta, \quad \tilde{u}_{k}(-\mathrm{i} \gamma)=(-\mathrm{i})^{k} u_{k}(\gamma)
$$

all terms are real if $x>r$ with positive $-\mathrm{i} \gamma$. Numerically, the uniform asymptotic expansion breaks down if $x$ comes close to $r$. But since it is analytic, one can expand it around the transitional point $x=r$ and obtain

$$
\begin{aligned}
\mathrm{e}^{\frac{\pi r}{2}} K_{\mathrm{i} r}\left(r-t r^{\frac{1}{3}}\right) \sim \pi\left(\left(\frac{2}{r}\right)^{\frac{1}{3}} A i(\right. & \left.-2^{\frac{1}{3}} t\right) \sum_{k=0}^{\infty}(-1)^{k} \frac{\tilde{A}_{k}(t)}{r^{\frac{2 k}{3}}} \\
& \left.+\left(\frac{4}{r}\right)^{\frac{1}{3}} A i^{\prime}\left(-2^{\frac{1}{3}} t\right) \sum_{k=0}^{\infty}(-1)^{k} \frac{\tilde{B}_{k}(t)}{r^{\frac{2 k}{3}}}\right), \quad t \text { small, }
\end{aligned}
$$

where the polynomials $\tilde{A}_{k}(t)$ and $\tilde{B}_{k}(t)$ are given in Olv52, eq. (2.42)]. Another useful asymptotic expansion in the transitional region is the Nicholson series MOS66, p. 145], Bal67, eq. (8)]

$$
\mathrm{e}^{\frac{\pi r}{2}} K_{\mathrm{i}\left(x-t x^{\frac{1}{3}}\right)}(x) \sim \pi\left(\frac{2}{x}\right)^{\frac{1}{3}} P(x, t) A i(Q(x, t)), \quad t \text { small, }
$$

where the functions $P(x, t)$ and $Q(x, t)$ are defined by

$$
P(x, t)=\sum_{k=0}^{\infty}\left(\frac{2}{x}\right)^{\frac{2 k}{3}} p_{k}\left(2^{\frac{1}{3}} t\right), \quad Q(x, t)=\sum_{k=0}^{\infty}\left(\frac{2}{x}\right)^{\frac{2 k}{3}} q_{k}\left(2^{\frac{1}{3}} t\right),
$$


and the polynomials $p_{k}(t)$ and $q_{k}(t)$ are given in [Sch54, p. 290]. Substituting asymptotic expansions of the Airy function in the uniform asymptotic expansion of the $K$-Bessel function leads to the Hankel series

$$
\begin{aligned}
\mathrm{e}^{\frac{\pi r}{2}} K_{\mathrm{i} r}(x) \sim \sqrt{2 \pi \gamma r}\left(\sin \left(\frac{2}{3}(-\xi)^{\frac{3}{2}}+\frac{\pi}{4}\right) \sum_{k=0}^{\infty} \frac{(-1)^{k}}{r^{2 k}} u_{2 k}(\gamma)\right. \\
\left.\quad+\cos \left(\frac{2}{3}(-\xi)^{\frac{3}{2}}+\frac{\pi}{4}\right) \sum_{k=0}^{\infty} \frac{(-1)^{k}}{r^{2 k+1}} u_{2 k+1}(\gamma)\right), \quad x \ll r,
\end{aligned}
$$

and the Debye series

$$
\mathrm{e}^{\frac{\pi r}{2}} K_{\mathrm{i} r}(x) \sim \sqrt{2 \pi(-\mathrm{i} \gamma) r}\left(\frac{1}{2} \exp \left(-\frac{2}{3} \xi^{\frac{2}{3}}\right) \sum_{k=0}^{\infty} \frac{1}{r^{k}} \tilde{u}_{k}(-\mathrm{i} \gamma)\right), \quad x \gg r
$$

see, e.g., Olv54 eqs. (2.14), (2.19)], AS65 eqs. (9.7.8), (9.3.10)], Bal67 eqs. (3), (5)], [CGS91, eqs. (A9), (A10)]. We numerically tested the given asymptotic expansions against each other - and the stationary phase algorithm in Hej92a - to find out their range of applicability and their accuracy. In this way, we found that by using the first five summands in the Hankel, the Debye and the Nicholson series, respectively, the $K$-Bessel function could be approximated with an accuracy of at least 10 digits for $r \approx 40000$ and all $x>0$.

\section{ACKNOWLEDGMENTS}

The encouraging advice of Professors Dennis A. Hejhal and Frank Steiner is gratefully acknowledged. Highest thanks are also due to Ralf Aurich, Jens Bolte, David Farmer, and Andreas Strömbergsson. For the stay in Professor Hejhal's group, where part of the work was done, the author was supported by the European Commission Research Training Network HPRN-CT-2000-00103. Currently, the author is supported by the Deutsche Forschungsgemeinschaft under the contract no. DFG Ste 241/16-1. The computations were run on the computers of the Universitäts-Rechenzentrum Ulm.

\section{REFERENCES}

[AS65] M. Abramowitz and I. A. Stegun, Handbook of Mathematical Functions, Dover, 1965. MR 34:8606

[ASD71] A. O. L. Atkin and H. P. F. Swinnerton-Dyer, Modular forms on noncongruence subgroups, Combinatorics (Proc. Sympos. Pure Math., Vol. XIX, Univ. California, Los Angeles, Calif., 1968), Amer. Math. Soc. (1971), 1-25. MR 49:2550

[Ave03] H. Avelin, On the deformation of cusp forms (Licentiate Thesis), UUDM report 2003:8 (Uppsala 2003).

[Bal66] C. B. Balogh, Uniform asymptotic expansions of the modified Bessel function of the third kind of large imaginary order, Bull. Amer. Math. Soc. 72 (1966), 40-43. MR 32:5942

[Bal67] C. B. Balogh, Asymptotic expansions of the modified Bessel function of the third kind of imaginary order, SIAM J. Appl. Math. 15 (1967), no. 5, 1315-1323. MR 36:5406

[Ber77] M. V. Berry, Regular and irregular semiclassical wavefunctions, J. Phys. A 10 (1977), 2083-2091. MR 58:8961

[BGGS92] E. Bogomolny, B. Georgeot, M.-J. Giannoni, and C. Schmit, Chaotic billiards generated by arithmetic groups, Phys. Rev. Lett. 69 (1992), 1477-1480. MR 93g:81025

[BLS96] E. Bogomolny, F. Leyvraz, and C. Schmit, Distribution of eigenvalues for the modular group, Comm. Math. Phys. 176 (1996), no. 3, 577-617. MR 97k:81052 
[Bol93] J. Bolte, Some studies on arithmetical chaos in classical and quantum mechanics, Int. J. Mod. Phys. B 7 (1993), 4451-4553. MR 94j:81049

[BSS92] J. Bolte, G. Steil, and F. Steiner, Arithmetical chaos and violation of universality in energy level statistics, Phys. Rev. Lett. 69 (1992), 2188-2191. MR 93f:81039

[Car71] P. Cartier, Some numerical computations relating to automorphic functions, Computers in Number Theory (A. O. L. Atkin and B. J. Birch, eds.), Academic Press, 1971, pp. 37-48. MR 47:3285

[Car78] P. Cartier, Analyse numérique d'un problème de valeurs propres a haute précision [application aux fonctions automorphes], preprint, IHES (1978).

[CFU57] C. Chester, B. Friedman, and F. Ursell, An extension of the method of steepest descents, Proc. Camb. Phil. Soc. 53 (1957), 599-611. MR 19:853a

[CGS91] A. Csordás, R. Graham, and P. Szépfalusy, Level statistics of a noncompact cosmological billiard, Phys. Rev. A 44 (1991), 1491-1499.

[GS82] V. V. Golovčanskiǐ and M. N. Smotrov, The first few eigenvalues of the Laplacian on the fundamental domain of the modular group, preprint, Far Eastern Scientific Center, Academy of Science USSR, Wladiwostok (1982) (Russian).

[GS84] V. V. Golovčanskiǐ and M. N. Smotrov, Calculation of first Fourier coefficients of eigenfunctions of the Laplace operator on the fundamental domain of a modular group, Numerical methods in algebra and analysis, Akad. Nauk SSSR, Dal. nevostochn. Nauchn. Tsentr, Vladivostok 85 (1984), 15-19 (Russian). MR 87i:11068

[GST02] A. Gil, J. Segura, and N. M. Temme, Computation of the modified Bessel function of the third kind of imaginary orders: Uniform Airy-type asymptotic expansion, CWI report MAS-R0205 (2002).

[HA93] D. A. Hejhal and S. Arno, On Fourier coefficients of Maass waveforms for PSL(2, Z), Math. Comp. 61 (1993), 245-267. MR 94a:11062

[Haa77] H. Haas, Numerische Berechnung der Eigenwerte der Differentialgleichung $-\Delta u=$ $\lambda y^{-2} u$ für ein unendliches Gebiet im $\mathbb{R}^{2}, 1977$, Diplomarbeit, Universität Heidelberg, Institut für Angewandte Mathematik.

[HB82] D. A. Hejhal and B. Berg, Some new results concerning eigenvalues of the nonEuclidean Laplacian for PSL $(2, \mathbb{Z})$, Tech. report 82-172, University of Minnesota, 1982.

[Hej81] D. A. Hejhal, Some observations concerning eigenvalues of the Laplacian and Dirichlet $L$-series, Recent Progress in Analytic Number Theory (H. Halberstam and C. Hooley, eds.), Academic Press, 1981, pp. 95-110. MR 82k:10005b

[Hej83] D. A. Hejhal, The Selberg trace formula for PSL(2, R), Lecture Notes in Math. 1001, Springer, 1983. MR 86e: 11040

[Hej91] D. A. Hejhal, Eigenvalues for the Laplacian for $\operatorname{PSL}(2, \mathbb{Z})$ : some new results and computational techniques, International Symposium in Memory of Hua Loo-Keng (S. Gong, Q. K. Lu, Y. Wang, and L. Yang, eds.), Springer, 1991, pp. 59-102. MR 92j:11048

[Hej92a] D. A. Hejhal, Eigenvalues of the Laplacian for Hecke triangle groups, Mem. Amer. Math. Soc. 469 (1992). MR 93f:11043

[Hej92b] D. A. Hejhal, On eigenvalues of the Laplacian for Hecke triangle groups, Zeta Functions in Geometry (N. Kurokawa and T. Sunada, eds.), vol. 21, Adv. Stud. Pure Math., 1992, pp. 359-408. MR 95f:11065

[Hej99] D. A. Hejhal, On eigenfunctions of the Laplacian for Hecke triangle groups, Emerging applications of number theory (D. A. Hejhal, J. Friedman, M. C. Gutzwiller, and A. M. Odlyzko, eds.), IMA Series No. 109, Springer, 1999, pp. 291-315. MR 2000f:11063

[HR92] D. A. Hejhal and B. Rackner, On the topography of Maass waveforms for PSL $(2, \mathbb{Z})$, Experiment. Math. 1 (1992), 275-305. MR 95f:11037

[HS01] D. A. Hejhal and A. Strömbergsson, On quantum chaos and Maass waveforms of CM-type, Found. Phys. 31 (2001), no. 3, 519-533. MR 2003k:81066

[Hun91] W. Huntebrinker, Numerische Bestimmung von Eigenwerten des Laplace-Operators auf hyperbolischen Räumen mit adaptiven Finite-Element-Methoden, Bonner Mathematische Schriften 225 (1991). MR 93f:65079

[Iwa95] H. Iwaniec, Introduction to the Spectral Theory of Automorphic Forms, Revista Matemática Iberoamericana, 1995. MR 96f:11078

[Kub73] T. Kubota, Elementary Theory of Eisenstein Series, Kodansha, Tokyo and Halsted Press, 1973. MR 55:2759 
[Maa49] H. Maaß, Über eine neue Art von nichtanalytischen automorphen Funktionen und die Bestimmung Dirichletscher Reihen durch Funktionalgleichungen, Math. Ann. 121 (1949), 141-183. MR 11:163c

[Maa64] H. Maaß, Lectures on Modular Functions of one Complex Variable, Tata Institute of Fundamental Research, 1964, Springer, Revised 1983. MR 85g:11034

[Miy89] T. Miyake, Modular forms, Springer, 1989. MR 90m:11062

[MOS66] W. Magnus, F. Oberhettinger, and R. P. Soni, Formulas and Theorems for the Special Functions of Mathematical Physics, Springer, 1966. MR 38:1291]

[Olv52] F. W. J. Olver, Some new asymptotic expansions for Bessel functions of large orders, Proc. Camb. Phil. Soc. 48 (1952), 414-427. MR 14:45b

[Olv54] F. W. J. Olver, The asymptotic expansion of Bessel functions of large order, Phil. Trans. A 247 (1954), 328-368. MR 16:696a

[Roe66] W. Roelcke, Das Eigenwertproblem der automorphen Formen in der hyperbolischen Ebene, Math. Ann. 167 (1966), 292-337 and 168 (1967), 261-324. MR 39:4386

[Sar95] P. Sarnak, Arithmetic quantum chaos, Israel Math. Conf. Proc. 8 (1995), 183-236. MR 96d:11059

[Sch54] W. Schöbe, Eine an die Nicholsonformel anschließende asymptotische Entwicklung für Zylinderfunktionen, Acta. Math. 92 (1954), 265-307. MR 16:696b

[Sch91] C. Schmit, Triangular billiards on the hyperbolic plane: Spectral properties, preprint, IPNO/TH 91-68 (1991).

[Sel56] A. Selberg, Harmonic analysis and discontinuous groups in weakly symmetric Riemannian spaces with applications to Dirichlet series, J. Indian Math. Soc. 20 (1956), 47-87. MR 19:531g

[SS02] B. Selander and A. Strömbergsson, Sextic coverings of genus two which are branched at three points, UUDM report 2002:16 (Uppsala 2002).

[Sta84] H. M. Stark, Fourier coefficients of Maass waveforms, Modular Forms (R. A. Rankin, ed.), Ellis Horwood, 1984, pp. 263-269. MR 87h:11128

[Ste92] G. Steil, Über die Eigenwerte des Laplaceoperators und der Heckeoperatoren für $\mathrm{SL}(2, \mathbb{Z})$, 1992, Diplomarbeit, Universität Hamburg, II. Institut für Theoretische Physik.

[Ste94] G. Steil, Eigenvalues of the Laplacian and of the Hecke operators for PSL(2, Z), DESY report 94-28 (Hamburg 1994).

[Ter85] A. Terras, Harmonic Analysis on Symmetric Spaces and Applications, vol. 1, Springer, 1985. MR 87f:22010

[Ven90] A. B. Venkov, Spectral Theory of Automorphic Functions and Its Applications, Kluwer Academic Publishers, 1990. MR 93a:11046

[Vig83] M.-F. Vignéras, Quelques remarques sur la conjecture $\lambda_{1} \geq \frac{1}{4}$, Séminaire de Théorie des Nombres (M.-J. Bertin, ed.), Birkhäuser, 1983, pp. 321-343. MR 85c:11049

[Wat44] G. N. Watson, A treatise on the theory of Bessel functions, Cambridge University Press, 1944. MR 6:64a

[Win88] A. M. Winkler, Cusp forms and Hecke groups, J. Reine Angew. Math. 386 (1988), 187-204. MR 90g:11067

Abteilung Theoretische Physik, Universität Ulm, 89069 Ulm, Germany

E-mail address: holger.then@physik.uni-ulm.de

$U R L:$ http://www.physik.uni-ulm.de/theo/qc/group.html 